
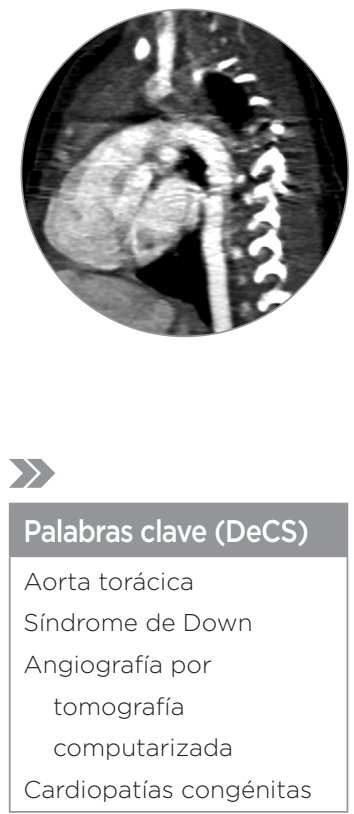

\section{Key words (MeSH)}

Aorta, thoracic

Down syndrome

Computed tomography

angiography

Heart defects, congenital

\title{
Interrupción de arco aórtico en síndrome de Down: presentación de un caso con revisión radiológica
}

\author{
Interrupted Aortic Arch with Down's Syndrome: A Case Report with \\ Radiological Review
}

\author{
Óscar Andrés Parada Duarte \\ María Fernanda Oviedo Lara ${ }^{2}$
} Andrés Mora Cáceres ${ }^{3}$

\begin{abstract}
Resumen
Introducción: La interrupción del arco aórtico (IAA) es una anomalía obstructiva, poco frecuente en la población en general, siendo diferente al momento de presentar concomitancia con otras anomalías cardiacas o síndromes genéticos, como síndrome de DiGeorge y síndrome de Down. Presentación de caso: Se trata de una recién nacida con facies características del síndrome de Down, diagnosticada con interrupción de arco aórtico tipo A, mediante angiografía por tomografía computarizada. Discusión: En la literatura se han descrito hasta el momento tres tipos de interrupción del arco aórtico (IAA), en los cuales se ha identificado como característica común un conducto arterioso persistente que permite la continuidad entre la arteria pulmonar y la aorta descendente. No existen informes de algoritmo diagnóstico para la detección de esta patología, pero sí se ha estudiado mediante ecocardiograma 2D, la angiografía por tomografía computarizada (TAC) y resonancia magnética (RM). Su tratamiento definitivo es quirúrgico. Conclusión: Se presenta un caso de la interrupción del arco aórtico con una revisión amplia de la literatura que se correlaciona con imágenes radiológicas, con el fin de generar conocimiento sobre dicha patología lo que permite un diagnóstico oportuno, fortalece la conducta médica y conduce a un mejor manejo de los recursos médicos.
\end{abstract}

\begin{abstract}
Summary
Introduction: Interrupted Aortic Arch (IAA) is an obstructive anomaly, with a rare frequency of isolated presentation in the general population, being different when it presents concomitance with other cardiac anomalies and / or genetic syndromes such as DiGeorge Syndrome and Down Syndrome. Case report: The case of a newborn with facies characteristic of Down Syndrome, diagnosed with Interrupted Aortic Arch type A, is presented through computed tomography angiography. Discussion: Three types of Interrupted Aortic Arch (IAA) have been described so far, in which a persistent arterial ductus that allows continuity between the pulmonary artery and the descending aorta has been identified as a common feature. There are no reports of diagnostic algorithm for the detection of this pathology, but it has been studied using 2D echocardiography, computed tomography angiography (CT) and magnetic resonance imaging (MRI). Its definitive treatment is surgical. Conclusion: A case report of the interruption of the aortic arch is presented with a comprehensive review of the literature that correlates with radiological images, to cause an impact on the generation of knowledge of said pathology, which allows for a timely diagnosis, strengthens our medical behavior. and leads to better management of medical resources.
\end{abstract}

\section{Introducción}

La interrupción del arco aórtico (IAA) es una mal-

Médico de la Universidad Nacional de Colombia, especialista en Radiología de la Universidad Militar de Colombia. Docente área clínica del programa de Medicina de la Universidad de Pamplona. Radiólogo de la Clínica Medical Duarte. Cúcuta, Colombia.

${ }^{2}$ Estudiante de noveno semestre de Medicina de la Universidad de Pamplona. Cúcuta, Colombia.

3Médico de la Universidad de Santander. Bucaramanga, Colombia. formación congénita poco frecuente, que representa la forma más grave en cuanto a anomalías obstructivas del arco aórtico (1), en la cual se ha perdido la continuidad luminal entre la aorta proximal y la aorta descendente. Se describió por primera vez en 1778 por Steidele (2), quien informó que el flujo hacia la aorta descendente se da por el ductus, el cual permanece permeable y brinda continuidad entre el tronco de la arteria pulmonar y la aorta distal. Tiene una incidencia de tres casos por millón de recién nacidos vivos, y representa el $1 \%$ de todas las anomalías cardiovasculares (3). Tiene una fuerte aso- ciación con cromosomopatías como 22q11 (síndrome de DiGeorge) con una incidencia aproximada de $25 \%$ (4), en la trisomía 21 (síndrome de Down) asociada a malformaciones cardiovasculares en el 44-50\% de los casos (5) y con las anomalías obstructivas del arco aórtico en aproximadamente 0,69 de 1000 nacidos vivos (6). Dada su baja incidencia se convierte en un tema poco estudiado, lo que hace necesario generar conocimientos claros frente a esta patología con el fin de realizar un diagnóstico oportuno. Se presenta el caso de un recién nacido con interrupción aórtica diagnosticada mediante angiografía por tomografía computarizada (TC). 


\section{Caso clínico}

Paciente femenina de 24 horas de nacida, de madre multípara. Al momento del parto tenía un peso de 3095 gr y talla de $49 \mathrm{~cm}$ y en el examen físico se evidenciaron facies características de síndrome de Down. La madre negó antecedentes de importancia. Se realizó un ecocardiograma 2D, del cual se recibió un informe escrito con resultado de hipoplasia del arco aórtico, del istmo aórtico con coartación aórtica; comunicación interventricular posterior amplia sin repercusión hemodinámica, conducto arterioso persistente, gran cortocircuito bidireccional sin repercusión hemodinámica; comunicación interauricular tipo ostium secundum sin repercusión hemodinámica. Después de evaluar el resultado, cardiopediatría decide hospitalizar a la paciente en la unidad de cuidado intensivo (UCI) pediátrica.

En las primeras horas de estancia hospitalaria la paciente desarrolla dificultad respiratoria y cianosis, por lo cual se le inicia manejo médico. Se le toma una angiografía por TC (angioTC), la cual, en su corte axial, confirma un arco aórtico hipoplásico (figura 1A) y una significativa dilatación de la arteria pulmonar (figura 1B) visibles también en los cortes sagitales y coronales (figuras 2 y 3 ). Además, en el corte sagital se visualiza persistencia del conducto arterioso y continuación de la arteria aorta descendente con la arteria pulmonar (figura 2A). Se confirma el diagnóstico de interrupción aórtica tipo A debido a la discontinuidad entre la aorta ascendente que se continúa con la arteria carótida derecha e izquierda, y la arteria pulmonar con significativa dilatación de aproximadamente $13 \mathrm{~mm}$ de diámetro (figura 2B). Se revisa la imagen en reconstrucción en 3D la cual confirma los hallazgos mencionados (figura 4). Tras el diagnóstico de dicha patología, la paciente se trata con prostaglandina E1 en su estancia en UCI pediátrica; sin embargo, la paciente fallece en su periodo neonatal.

\section{Discusión}

La interrupción del arco aórtico es una de las formas más graves y la anomalía congénita más rara en el espectro de lesiones obstructivas del arco aórtico, definida como pérdida de la continuidad anatómica entre aorta ascendente y descendente y considerada una forma extrema de coartación (1-3). El flujo hacia la aorta descendente lo proporciona el conducto, que permanece permeable y brindando continuidad entre el tronco de la arteria pulmonar y la aorta distal. A pesar de esto, la sangre que llega al abdomen y miembros inferiores está parcialmente oxigenada. Los pacientes evolucionan con insuficiencia cardiaca congestiva grave, tendencia al shock $\mathrm{y}$, en caso de no realizarse un tratamiento oportuno, suelen fallecer en el periodo neonatal (3).

Hoy en día se reconocen diferentes formas de manifestación de la IAA y se han descrito distintas clasificaciones, por ejemplo, la de Celoria y Patton (1959), que la clasifican según el sitio de la interrupción: Tipo A, la interrupción se encuentra distal a la arteria subclavia izquierda; tipo B, se ubica distal a la arteria carótida común izquierda, por lo tanto, la arteria subclavia izquierda surge distal a la interrupción de la aorta descendente; y tipo $\mathrm{C}$, que es excepcionalmente raro, está entre el tronco arterial braquiocefálico (arteria innominada) y la arteria carótida común izquierda (1).

Epidemiológicamente, la IAA se encuentra en alrededor de 3 casos por millón de nacidos vivos y representa el $1 \%$ de todas las anomalías cardiovasculares (3). Pero su incidencia cambia por su frecuente asociación con otras anomalías cardiovasculares (conducto persistente, defectos en el septum ventricular, comunicaciones interventriculares, etc.), así como a enfermedades o síndromes genéticos, como el síndrome de DiGeorge y el de Down, con una frecuencia del $25 \%$ y del 0,069 \%, respectivamente (4-6). Así mismo, según dicha clasificación, la tipo B se presenta en un $70 \%$, mientras que la tipo A, en un $28 \%$ y la tipo C, en un $1 \%$ (1).
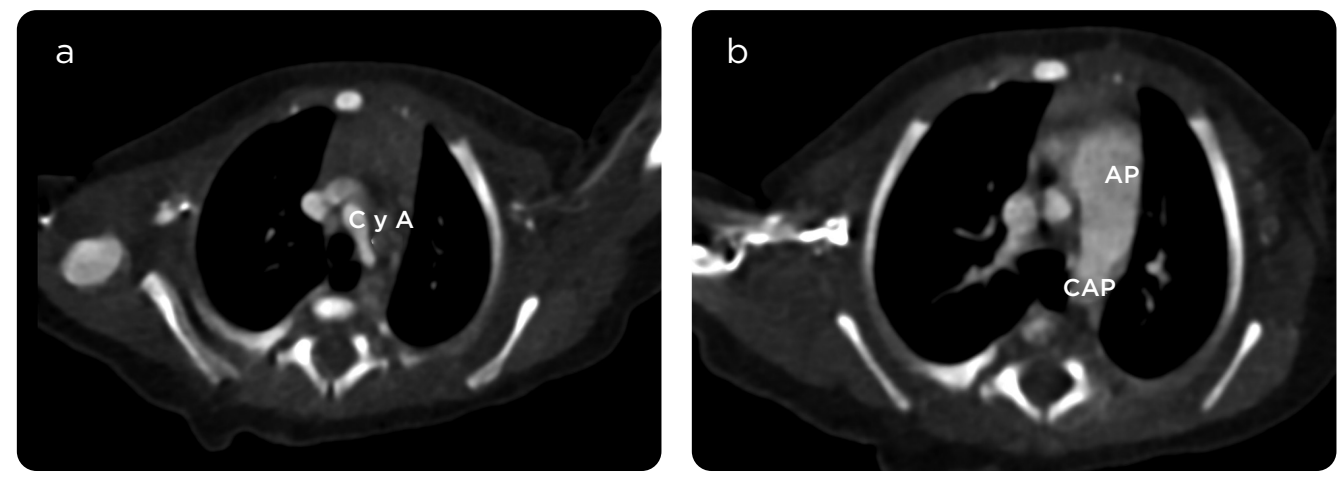

Figura 1. AngioTC, a) corte axial: leve hipoplasia del cayado aórtico (CyA). b) Significativa dilatación de la arteria pulmonar (AP) y conducto arterioso persistente (CAP, PDA por las iniciales en inglés de patent ductus arteriosus) sin dilatación.

Figura 2. AngioTC, a) corte sagital: arteria pulmonar (AP) con significativa dilatación de $13 \mathrm{~mm}$ de diámetro, conducto arterioso persistente (CAP) de $6 \mathrm{~mm}$ de diámetro y el origen de la arteria aorta descendente desde el conducto arterioso, con evidente continuidad. b) Se observa la discontinuidad y la diferencia de origen entre la arteria aorta ascendente (AA) y la arteria pulmonar.
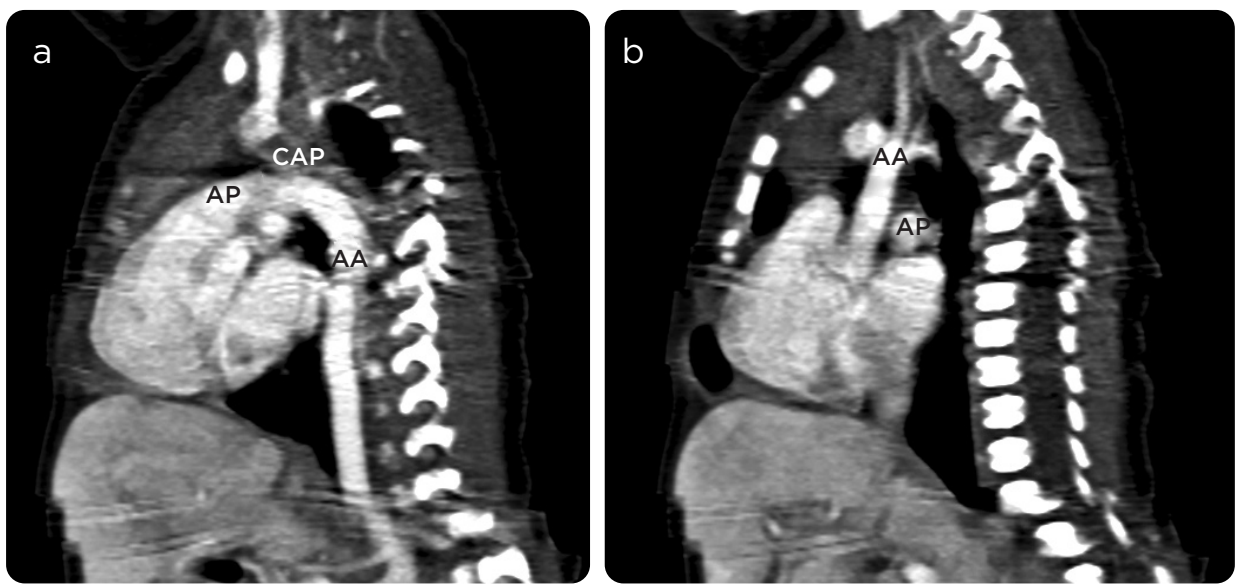


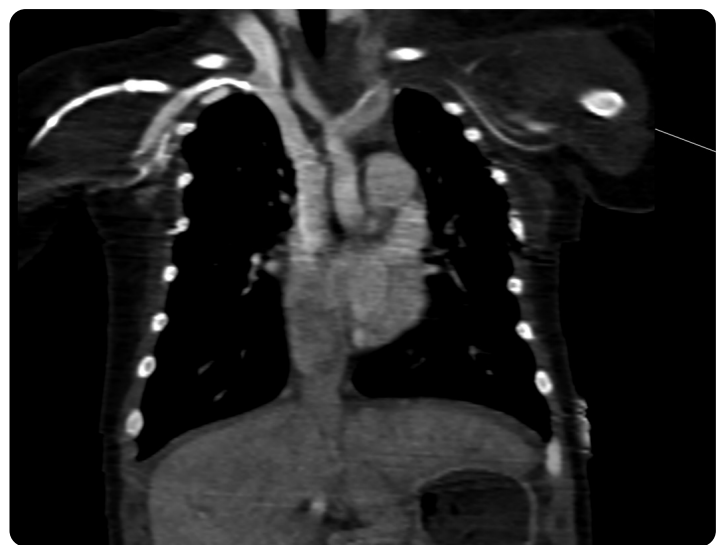

Figura 3. AngioTC, corte coronal: origen no concordante entre la aorta ascendente y la arteria pulmonar, la cual se encuentra significativamente dilatada.
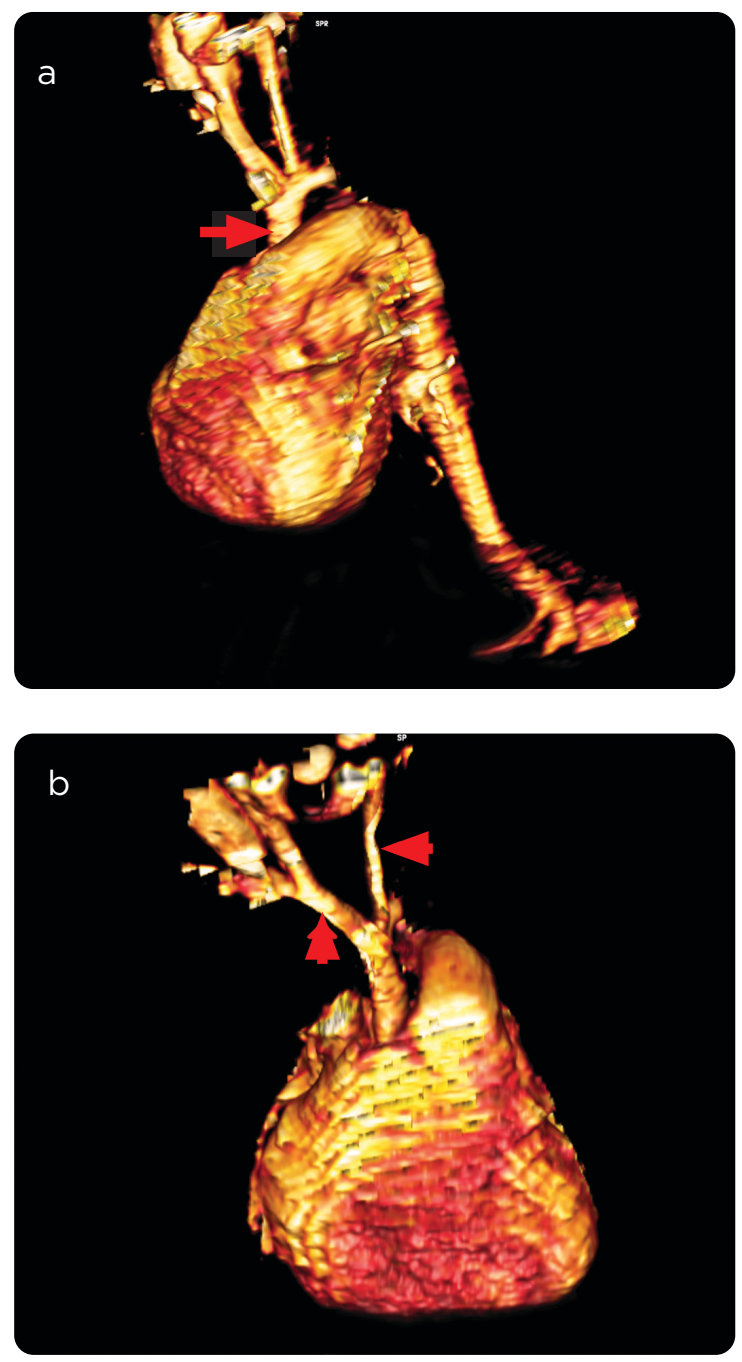

Figura 4. Reconstrucción 3D angioTC. Vista lateral y anterior: se observa la interrupción aórtica tipo A acompañada de una dilatación significativa de la arteria pulmonar de $13 \mathrm{~mm}$ de diámetro (AP). La aorta ascendente (flecha grande) continúa como las arterias carótidas comunes derecha (punta de flecha pequeña) e izquierda (punta de flecha grande). Un conducto arterioso persistente (CAP) de $6 \mathrm{~mm}$ de diámetro da origen a la aorta descendente.
Embriológicamente, en condiciones normales, la arteria torácica se forma mediante la creación de seis arcos aórticos bilaterales y las séptimas arterias intersegmentarias, con la involución de varios segmentos de estos. Así, de acuerdo con el nivel de fallo en la regresión se puede clasificar en IAA tipo A, que se forma por la regresión anormal del cuarto arco izquierdo tardío en el desarrollo, después de que la arteria subclavia izquierda ha ascendido a su posición normal (7).

Existen diversos mecanismos para diagnosticar esta patología sin que exista un algoritmo específico, entre ellos los protocolos o guías específicos de cada institución, adaptados a los recursos disponibles. En el caso descrito se realizó un ecocardiograma 2D; sin embargo, este método es limitado, pues no permite observar adecuadamente la continuación de la arteria pulmonar principal a través del conducto arterioso a la aorta descendente (arco ductal), el cual puede parecerse a un verdadero arco aórtico. No obstante, es posible diferenciarlo por la ausencia del origen de los vasos braquiocefálicos. Además, la anatomía cardiaca en más de dos tercios de los casos se caracteriza por un corazón biventricular con conexiones normales y una comunicación interventricular grande (1).

La ecocardiografía no diferencia un caso de IAA de otro en el que coexistan coartación aórtica grave y un arco aórtico hipoplásico, por lo que es necesario realizar exámenes complementarios como TC o RM. Estas técnicas demuestran las características morfológicas de la IAA y los posibles hallazgos complejos asociados, debido a sus capacidades multiplanares, que facilitan la comprensión de la anomalía y sus relaciones anatómicas. Sin embargo, la TC tiene más ventajas, como menor tiempo de realización con menos requerimientos de sedación y mayor resolución de las imágenes con vista simultánea de la vía aérea (8).

Los pacientes con IAA se someten a cirugía cardiaca durante el primer año de vida y tienen una tasa de supervivencia global a los 16 años de alrededor del 59-70 \%. Se ha demostrado que la administración de la terapia con prostaglandina E1 en el período neonatal, para mantener abierto el conducto arterioso, mejora la supervivencia. El objetivo del tratamiento es restablecer la continuidad de la aorta; sin embargo, el abordaje quirúrgico es controvertido. El más estudiado consiste en reconstrucción mediante una anastomosis de extremo a extremo, creación de un colgajo subclavio izquierdo, reducción carotidea y colocación de un injerto de interposición. La mortalidad postoperatoria está entre el $15 \%$ y el $20 \%$ (4).

\section{Conclusión}

La literatura mundial describe la IAA como una cardiopatía congénita infrecuente, con pocos informes de casos y una prevalencia baja, que se diagnostica de forma concomitante con otras patologías, principalmente asociada a síndromes congénitos. Al informar este caso se insiste en la necesidad de profundizar en el conocimiento de dicha patología, para poder realizar diagnóstico de interrupción del arco aórtico tipo A, mediante las herramientas disponibles como la ecocardiografía $2 \mathrm{D}$ y la angiografía por TC. Se presentan imágenes radiológicas como aporte al desarrollo de un diagnóstico oportuno en las instituciones médicas del departamento, una región en la cual no se encuentran informes de la patología ni datos acerca de casos confirmados.

\section{Referencias}

1. Hofbeck M, Deeg K, Rupprecht T. Interruption of the aortic arch. En: Hofbeck M Deeg K, Rupprecht T. Doppler echocardiography in infancy and childhood. Suiza: Springer; 2017. pp. 297-308. 
2. Garcés Garcés J, Cortez Flores P, Gordillo Tobar L, et al. A case never reported before: bilateral type A interruption in a double aortic arch without persistent ductus arteriosus. Cardiovasc Metabolic Sci. 2019;30(2):60-5.

3. Haberman D, Gurfinkel E, Beresñak A, et al. Anomalías congénitas de la arteria aorta. Evaluación mediante tomografía computada multicorte en neonatos y niños. Rev Arg Radiol. 2009;73(3):284-5.

4. Khaladkar S, Chaudhary K, Kuber R, et al. Interrupted aortic arch by multi-detector computed tomography angiography: A case report with radiological review. CHRISMED J Health Res. 2016;3(1):83-6.

5. Freeman SB, Taft LF, Dooley KJ, et al. Population-based study of congenital heart defects in Down syndrome. Am J Med Genet. 1998;80(3):213-7.

6. Torfs C, Christianson R. Anomalies in Down syndrome individuals in a large population-based registry. Am J Med Genet. 1998;77(5):431-8.

7. Reardon MJ, Hallman GL, Cooley DA. Interrupted aortic arch: Brief review and summary of an eighteen-year experience. Tex Heart Inst J. 1984;11(3):250-9.

8. Kimura-Hayama ET, Meléndez G, Mendizábal AL, et al. Uncommon congenital and acquired aortic diseases: role of multidetector CT angiography. Radiographics. 2010;30(1):79-98.

\section{Correspondencia}

María Fernanda Oviedo Lara

Av. 12E \# 8N-08, apartamento 101

Barrio Ciudad Jardín

San José de Cúcuta, Norte de Santander, Colombia

moviedolara@gmail.com

Recibido para evaluación: 28 de octubre de 2019

Aceptado para publicación: 1 de diciembre de 2019 